\title{
Observations of enhanced nonlinear instability in the surface reflection of internal tides
}

\author{
Xiaohui Xie, ${ }^{1}$ Xiaodong Shang, ${ }^{1}$ Hans van Haren, ${ }^{2}$ and Guiying Chen ${ }^{1}$ \\ Received 4 February 2013; revised 4 March 2013; accepted 5 March 2013; published 29 April 2013.
}

[1] Enhanced vertically standing waves formed by the superposition of two upward and downward going neardiurnal $\left(D_{1}\right)$ waves are observed during one semidiurnal $\left(D_{2}\right)$ spring tide in an approximately 75 day long velocity record from the northeastern South China Sea. Bicoherence estimates suggest that the enhanced $D_{1}$ waves are likely due to nonlinear parametric subharmonic instability of $D_{2}$ internal tides. The timescale for energy growth by an order of magnitude is about 2.5 days for these waves. In addition to subharmonics, higher harmonics $D_{4}\left(=D_{2}+D_{2}\right)$ and a mean flow are generated by a different nonlinear interaction during the same $D_{2}$ spring tide. The separation of coherent from incoherent internal tidal signals and a rotary spectral decomposition in the vertical direction reveal that $D_{2}$ waves with opposite vertical propagation directions in the region of internal tide reflection from the surface may be responsible for the pronounced nonlinear instability. Citation: Xie, X., X. Shang, H. van Haren, and G. Chen (2013), Observations of enhanced nonlinear instability in the surface reflection of internal tides, Geophys. Res. Lett., 40, 1580-1586, doi:10.1002/grl.50322.

\section{Introduction}

[2] It is well known that semidiurnal internal tides $\left(\mathrm{IT}_{D 2}\right.$, internal waves with semidiurnal frequency $D_{2}$ ) dominate the internal wave spectrum in the ocean. Many observations and numerical models have suggested that $\mathrm{IT}_{D 2}$ can transfer their energy to a series of higher harmonics (e.g., $D_{4}, D_{6}$ ) via nonlinear (superharmonic) instability [e.g., van Haren et al., 2002; Lamb, 2004; Xie et al., 2008]. On the other hand, they can also lose energy to waves with frequencies near the diurnal frequency $D_{1}\left(=D_{2} / 2\right)$ and higher vertical wave number $k_{z}$ through parametric subharmonic instability (PSI) [e.g., Hibiya et al., 2002; van Haren, 2005; Alford et al., 2007]. Although in theory the classic PSI is thought to be a weakly nonlinear process [McComas and Bretherton, 1977], several recent numerical models [Gerkema et al., 2006; Korobov and Lamb, 2008] and field observations [Carter and Gregg, 2006; Xie et al., 2011] suggest that PSI of IT in the ocean may turn into a rapid, strong instability.

[3] Two regions where pronounced nonlinear instability may occur are suggested by numerical models and laboratory

\footnotetext{
${ }^{1}$ State Key Laboratory of Tropical Oceanography, South China Sea Institute of Oceanology, Chinese Academy of Sciences, Guangzhou, China.

${ }^{2}$ Royal Netherlands Institute for Sea Research (NIOZ), Den Burg, Netherlands.

Corresponding author: X. Shang, State Key Laboratory of Tropical Oceanography, South China Sea Institute of Oceanology, Chinese Academy of Sciences, Guangzhou 510301, China. (xdshang@scsio.ac.cn)

(C)2013. American Geophysical Union. All Rights Reserved. 0094-8276/13/10.1002/grl.50322
}

experiments: one is the boundary where internal wave beams are reflected [Javam et al., 2000; Mercier et al., 2012] and the other is the region where two beams intersect [Teoh et al., 1997; Korobov and Lamb, 2008]. In the ocean, few observations exist on nonlinear effects in the above mentioned two regions. Although IT can be modeled by a purely horizontally propagating (vertical) mode in a flat-bottomed ocean [Leblond and Mysak, 1978], this model is destroyed when the water depth varies. Then, one of the downward and upward propagations will be dominant. So in a real ocean with varying water depth, IT will always be a vertical traveling wave. In this paper, moored current measurements in the northeastern South China Sea (SCS) are used to investigate nonlinear effects between two oppositely propagating internal tidal waves in the vertical direction in the near-surface reflection area of IT. Our observations suggest that PSI from $\mathrm{IT}_{D 2}$ can be rapidly enhanced in the reflection region. A mean flow caused by the nonlinear interaction between these two waves is also observed.

\section{Data and Methods}

[4] Ocean currents are evaluated from one mooring deployed at $20.58^{\circ} \mathrm{N}, 118.41^{\circ} \mathrm{E}$, where the water depth is $2474 \mathrm{~m}$. The mooring consisted of an upward looking $75 \mathrm{kHz}$ acoustic Doppler current profiler (ADCP) at $\sim 450 \mathrm{~m}$ (data from 436 to $52 \mathrm{~m}, 16 \mathrm{~m}$ bins) and five Aanderaa current meters at 800 , $1000,1500,2000$, and $2300 \mathrm{~m}$. In this paper, the ADCP data and current meter data from 2000 and $2300 \mathrm{~m}$ are used. The mooring period was from August 2000 to March 2001. Unfortunately, the ADCP worked only during the first 75 days and current meters at 800,1000 , and $1500 \mathrm{~m}$ gave false data due to some incorrect settings before deployment. The sampling rates of $\mathrm{ADCP}$ and current meters were once per 15 and $30 \mathrm{~min}$, respectively. Data of the two current meters at 2000 and $2300 \mathrm{~m}$ are linearly interpolated onto the $15 \mathrm{~min}$ sample interval to match those of the ADCP data.

[5] In the SCS, diurnal $\left(D_{1}\right)$ internal tides $\left(\mathrm{IT}_{D 1}\right)$ are often dominant in the internal wave spectrum [Lien et al., 2005; Xie et al., 2009]. For a 75 day long record, one can easily separate inertial (frequency: $f=2 \pi /(34 \mathrm{~h})$ ), $D_{1}$, and $D_{2}$ motions. Using a second-order Butterworth filter, all current data are band-pass-filtered to extract the $D_{1}$ and $D_{2}$ components, using filter bounds $\left[0.9 O_{1}, 1.1 K_{1}\right]$ and $\left[0.86 M_{2}\right.$, $\left.1.14 M_{2}\right]\left(O_{1}, K_{1}\right.$, and $M_{2}$ are the primary tidal frequencies in the $D_{1}$ and $D_{2}$ bands), respectively. The band-passed $D_{1}$ and $D_{2}$ motions are further decomposed into coherent and incoherent components by applying a sharply harmonic filter [van Haren, 2004]. The coherent component containing barotropic tides and coherent IT (hereafter referred to as CIT) can be obtained by 

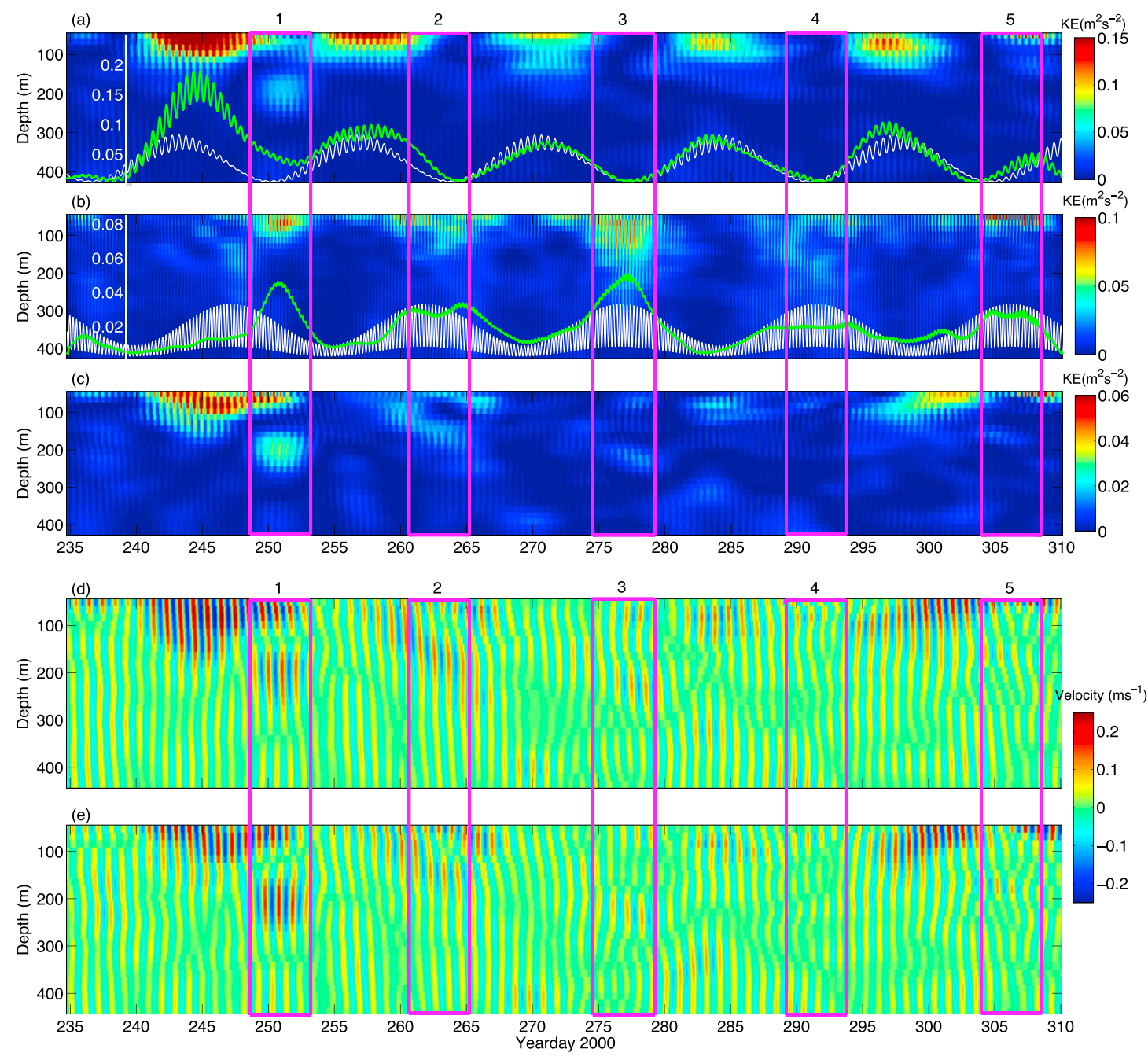

Figure 1. Time series of kinetic energy $(K E)$ and velocity from the ADCP data. (a) $K E_{D 1}$. (b) $K E_{D 2}$. (c) $K E$ of diurnal ICIT $\left(K E_{i c D 1}\right)$. (d) Zonal and (e) meridional velocities of $\operatorname{ICIT}_{D 1}$. The green and white curves in Figure 1a are $K E_{D 1}$ and $K E_{O 1+K 1}$ averaged above $120 \mathrm{~m}$, respectively. The green and white curves in Figure $1 \mathrm{~b}$ are $K E_{D 2}$ and $K E_{M 2+S 2}$ averaged above $120 \mathrm{~m}$, respectively. The white vertical scale in Figures $1 \mathrm{a}$ and $1 \mathrm{~b}$ indicates the magnitude of the green and white curves. In all panels, the purple rectangles indicate the periods of SSTs. The SSTs are numbered. Note the different color scales in Figures 1a, 1b, and 1c.

$$
\begin{gathered}
u_{c}=\sum_{n} U_{n} \cos \left(\omega_{n} t+\varphi_{n}\right) \\
\omega_{n}=Q_{1}, O_{1}, K_{1}\left(=2 N_{2}, M_{2}, S_{2} \text { for } D_{2} \text { band }\right),
\end{gathered}
$$

where $U_{n}$ is the amplitude of the $n$th constituent and $\varphi_{n}$ is its phase, similar for meridional component $v_{c}$ [van Haren, 2004]. The incoherent IT (hereafter referred to as ICIT) component is then defined as

$$
u_{i c}=u_{\text {Bandpass }}-u_{c},
$$

similar for the $v$ component. In equation (1), six frequencies are chosen, which all have a signal-to-noise ratio larger than 1.5 .

\section{Results}

\subsection{Diurnal ICIT}

[6] Figures $1 \mathrm{a}$ and $1 \mathrm{~b}$ show the time series of $D_{1}$ and $D_{2}$ kinetic energy, actually current variance, $K E_{D 1}$ and $K E_{D 2}$ $\left(K E=u^{2}+v^{2}\right)$, respectively. $K E_{D 1}$ and $K E_{D 2}$ values in the upper $120 \mathrm{~m}$ show approximate fortnightly spring-neap cycles. The diurnal 14 day cycle is induced by the superposition of $O_{1}$ and $K_{1}$ (Figure 1a), while the semidiurnal 14 day cycle is mainly induced by the superposition of $M_{2}$ and $S_{2}$ (Figure 1b). However, there is a significant variation in semidiurnal spring tides (SSTs). Especially, the largest $K E_{D 2}$ around the first SST (SST-1) appear on yearday 250, having a significant shift with respect to the peak of coherent $M_{2}+S_{2}$ signals. Hereafter, SSTs (marked by rectangles in Figure 1) refer to the periods with the largest $K E_{D 2}$ averaged over all observation depths above $120 \mathrm{~m}$ around the times of spring tide induced by coherent $M_{2}+S_{2}$ signals. The above shift is partly due to the modulation of other coherent constituents such as $2 N_{2}$. On the other hand, the shift may also be due to the varying background condition, which can generate incoherent components to destroy the 14 day spring-neap cycle [van Haren, 2004]. This is also likely to be one of the reasons for the generation of strong ICIT $_{D 1}$ around yearday 245 (Figure 1c). Noticeably, a relatively weak growth of $K E_{D 1}$ occurs near $200 \mathrm{~m}$ on yeardays 249 

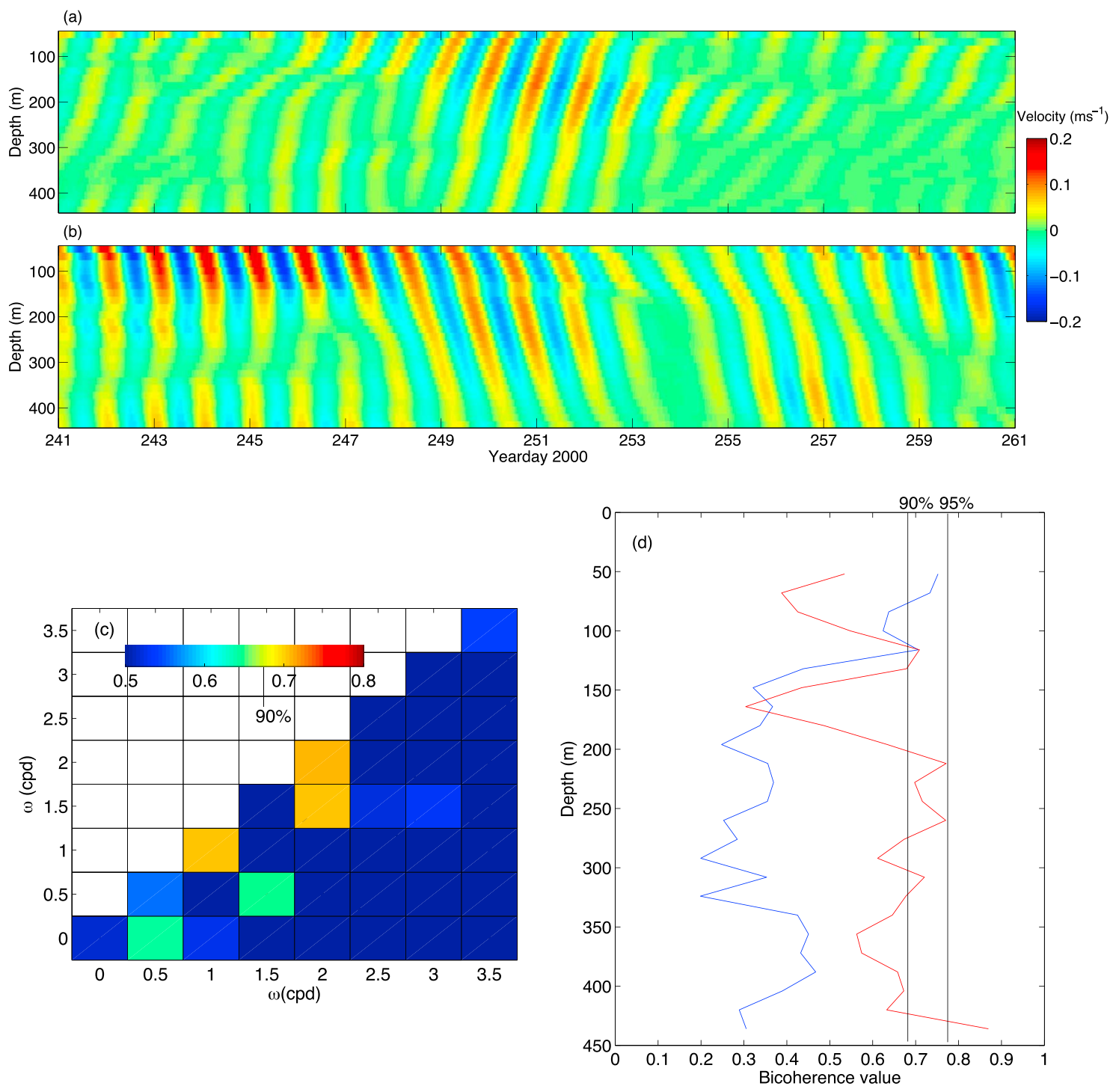

Figure 2. (a) $\mathrm{CW}$ and (b) ACW motions of $\mathrm{ICIT}_{D 1}$ from the ADCP data on yeardays 241 to 260. (c) Average bicoherence of $u$ and $v$ at $116 \mathrm{~m}$ during SST-1. Note that all values less than 0.5 are set to 0.5 for clarity. (d) Bicoherence values around $[1,1] \mathrm{cpd}$ (blue) and [2, 2] cpd (red) are plotted as a function of depth. The vertical lines give $90 \%$ and $95 \%$ significance levels.

to 252 corresponding to diurnal neap tide (Figures 1a and 1b). After removing the coherent $O_{1}+K_{1}+Q_{1}$ component, the $D_{1} 14$ day cycle disappears (Figure 1c). Enhanced $\operatorname{ICIT}_{D 1}$ only occur during some SSTs. Specifically during SST-1, ICIT $_{D 1}$ near $200 \mathrm{~m}$ and $D_{2}$ motions above $120 \mathrm{~m}$ are synchronously enhanced and weakened (Figure 1b). In both the zonal and meridional incoherent velocity fields of $D_{1}$ (Figures $1 \mathrm{~d}$ and $1 \mathrm{e}$ ), the enhanced $D_{1}$ motions show large $k_{z}(\approx 2 \pi / 300 \mathrm{~m})$. Furthermore, a checkerboard (vertically standing) pattern is clearly identified on yeardays 249 to 252, which indicates that the signals are the sum of upward and downward propagating waves. During other SSTs, signals with high $k_{z}(>2 \pi / 300 \mathrm{~m})$ are also observed, but no clear checkerboard pattern is found. In contrast, during $D_{1}$ springs $\mathrm{ICIT}_{D 1}$ show relatively low $k_{z}(<2 \pi / 300 \mathrm{~m})$.

[7] In order to confirm the bidirectional propagation of $\operatorname{ICIT}_{D 1}$ during SST-1, incoherent velocity of $D_{1}$ is decomposed into clockwise (CW) and anticlockwise (ACW) rotary motions with depth, which represent upward and downward phase propagation, respectively [Leaman and Sanford, 1975]. The decomposed velocity fields are shown in Figure 2. As expected, both upward (CW) and downward $(\mathrm{ACW})$ traveling signals with comparable amplitudes are clearly identified on yeardays 249 to 252 (Figures $2 \mathrm{a}$ and $2 \mathrm{~b}$ ). In contrast, ICIT $_{D 1}$ during diurnal springs (yeardays 241-246 and 255-260) are dominated by downward phase propagation.

\subsection{Bicoherence Spectra}

[8] In section 3.1, we show that the features of $\operatorname{ICIT}_{D 1}$ observed during SST-1 agree well with those of subharmonics as expected from PSI theory [McComas and Bretherton, 1977]. In order to provide evidence of near-diurnal subharmonic energy transfer, bicoherence (normalized bispectrum) is computed following Carter and Gregg [2006]. Because CIT $_{D 1}$ may contaminate this spectral estimation, coherent $O_{1}+K_{1}+$ $Q_{1}$ signals are removed from the raw velocity record before computing bicoherence. Here, only a 6 day long (yeardays 247-252) velocity record around SST-1 is used for the estimate. The 6 day long time series is divided into five segments 
with $50 \%$ overlap. Each 2 day time series segment is smoothed by a Kaiser-Bessel window, yielding a spectral estimation with $\sim 10$ degrees of freedom [Emery and Thomson, 2001].

[9] Figures 2c and 2d show averaged bicoherence of $u$ and $v$ velocity components. The bicoherence value at a particular frequency pair $\left[\omega_{1}, \omega_{2}\right]$ represents the strength of the triad wave-wave interaction at frequencies $\omega_{1}, \omega_{2}$, and $\omega_{1}+\omega_{2}$. Analysis at $116 \mathrm{~m}$ shows significant bicoherence around the frequency pair $[1,1] \mathrm{cpd}$ (Figure 2c), which is evidence for near-diurnal PSI energy transfer of $D_{2}$ waves. Significant bicoherence also appears around $[2,2] \mathrm{cpd}$, suggesting that quarter-diurnal $\left(D_{4}\right)$ waves, which are also enhanced during SST-1 (see Figure 3g), may be generated by self-nonlinear interaction of $D_{2}$ waves, namely $D_{2}+D_{2}=D_{4}$. For subharmonic instability of $D_{2}$ waves, significant bicoherence results at the $90 \%$ level only appear above $120 \mathrm{~m}$ (Figure $2 \mathrm{~d}$ ). This is consistent with the depth range with the largest $K E_{D 2}$ (see Figure 1b). However, large bicoherence values around $[2,2]$ cpd mostly appear below $200 \mathrm{~m}$ (Figure 2d).

\subsection{Bidirectional Propagation of $D_{2}$ Waves}

[10] In sections 3.1 and 3.2, it was shown that $\operatorname{ICIT}_{D 1}$ with a checkerboard pattern are likely generated by PSI of $D_{2}$ waves. Although we cannot confirm that other enhanced $\mathrm{ICIT}_{D 1}$, such as the weaker signals observed during SST-2, are also excited via PSI of $D_{2}$ waves, these signals are much weaker than those observed during SST-1. To explore the possible reason, primary waves $\left(D_{2}\right)$ are investigated. Since IT mainly propagate westward in the northeastern SCS [e.g., Lien et al., 2005], zonal velocities of $D_{2}$ around three SSTs (SST-1, SST-2, and SST-5) are presented. Velocity signals at different SSTs reveal large differences (Figures 3a and 4a). During SST-1 and SST-2, no clear phase propagation is observed above $400 \mathrm{~m}$, while signals during SST-5 show marked upward phase propagation (Figure 4a). During SST-2, velocities at small $(50-400 \mathrm{~m})$ and large (2000 and $2300 \mathrm{~m}$ ) depths are often out of phase, implying the dominance of local vertical mode-1. Although out-ofphase shallow-deep flows also appear during SST-1 and SST-5, higher-mode $D_{2}$ waves can be clearly identified. The higher-mode $D_{2}$ waves are primarily present in the form of ICIT because of their significant phase variation in the vertical direction (Figures $3 \mathrm{c}$ and $4 \mathrm{c}$ ).

[11] Around SST-1, the phase of $\mathrm{ICIT}_{D 2}+\mathrm{CIT}_{2 N 2}$ indicates downward phase propagation. On the contrary, $\mathrm{CIT}_{M 2+S 2}$ are dominated by waves with low $k_{z}$, showing upward phase propagation (Figure $3 \mathrm{~b}$ ). As coherent $2 \mathrm{~N}_{2}$ signals are weak, $\mathrm{ICIT}_{D 2}+\mathrm{CIT}_{2 N 2}$ is hereafter referred as $\mathrm{ICIT}_{D 2}$. At the same SST, the bidirectional vertical propagation of $\mathrm{IT}_{D 2}$ can also be clearly identified when $D_{2}$ currents are decomposed into $\mathrm{CW}$ and $\mathrm{ACW}$ rotary motions (Figures $3 \mathrm{~d}$ and $3 \mathrm{e}$ ). ICIT $_{D 2}$ with significant downward phase propagation are not found during SST-2 and SST-5 (Figure 4c), neither during SST-3 and SST-4 (not shown). Since during these SSTs CIT $_{M 2+S 2}$ with larger amplitude propagate upward (Figures $4 \mathrm{~b}$ and $4 \mathrm{c}$ ), CW rotary motions often dominate over ACW rotary motions (Figures $4 \mathrm{~d}$ and 4e). Furthermore, few events are observed with significant downward phase propagation in ACW signals during these periods. Thus, during SST-1, strong subharmonics may be generated via interaction between two vertically oppositely traveling $D_{2}$ waves. Their rapid energy growth is associated with the amplitude and phase of the two $D_{2}$ waves. This is because on one hand during other SSTs, near-diurnal subharmonics are not effectively enhanced due to weak amplitude or absence of downward (phase) propagating waves. On the other hand, the bidirectional propagation of $D_{2}$ waves also occurs on yeardays 246 to 248 (Figures 3b, $3 \mathrm{c}, 3 \mathrm{~d}$, and $3 \mathrm{e}$ ), during which upward propagating $\mathrm{CIT}_{M 2+S 2}$ and downward propagating ICIT $_{D 2}$ above $120 \mathrm{~m}$ are nearly out of phase, but there are no enhanced subharmonics. In contrast, when strong subharmonics occur on yeardays 249 to 252 , two waves are approximately in phase above $120 \mathrm{~m}$ where a strong interaction at $D_{1}+D_{1}=D_{2}$ is likely to occur (see Figure 2d).

[12] For the enhanced near-diurnal subharmonics on yeardays 249 to 252 , the timescale of their energy growth $K E /(d K E / d t)$, where subharmonic $K E$ is averaged over half a vertical wavelength (150-300 m; see Figures $1 \mathrm{~d}$ and $1 \mathrm{e})$, is estimated to be about 2.5 days (Figure $3 \mathrm{f}$ ). This means that the energy increases by an order of magnitude in about 2.5 days. This characteristic timescale is roughly consistent with that predicted by the numerical model of Gerkema et al. [2006] and that found in the field observations of MacKinnon et al. [2013] near the critical latitude of $28.8^{\circ}$ where the most effective PSI process is expected.

\subsection{Higher Harmonic and Mean Flow}

[13] In section 3.3, it was shown that rapidly growing neardiurnal subharmonics are associated with the bidirectional (vertical) propagation of $\mathrm{IT}_{D 2}$. Bidirectional propagation of IT near the surface may be due to the surface reflection of IT. Using small-amplitude expansion, Tabaei et al. [2005] theoretically discussed nonlinear effects in internal wave reflection from a boundary. They show that nonlinear interaction becomes pronounced in the reflection region where two IT beams meet. Thereby, a series of higher harmonics and a mean flow are generated. Recent laboratory experiments by Mercier et al. [2012] verify the generation of higher harmonics and a mean flow at the location where an IT beam reflects from the surface. To investigate whether these two motions are also generated when two oppositely traveling $D_{2}$ waves interact, the first higher harmonic $\left(D_{4}\right)$ and subinertial velocities are extracted from the present data. As expected, $D_{4}$ waves are enhanced around SST-1 (Figure 3g). The starting time of the increase is around yearday 246, when the vertical propagation direction of $\operatorname{ICIT}_{D 2}$ is opposite to that of $\mathrm{CIT}_{M 2+S 2}$ (Figures $3 \mathrm{~b}$ and $3 \mathrm{c}$ ). Meanwhile, a westward (negative) mean flow restricted to the upper layer emerges from the background eastward flow (Figure $3 \mathrm{~h}$ ). Since IT in our observation area are expected to travel westward [e.g., Lien et al., 2005], the direction of wave propagation is the same as that of the mean flow.

[14] The westward mean flow and elevation of $D_{4}$ waves, along with strong subharmonics (Figure 3f), disappear around yearday 252. Interestingly, the depth ranges where $K E_{D 4}$ is elevated are different in different periods (Figure $3 \mathrm{~g}$ ). On yeardays 246 to $248, D_{4}$ waves are enhanced only above $100 \mathrm{~m}$, where $D_{2}$ velocity is small (Figure 3a). After yearday 248 , the near-surface elevation of $K E_{D 4}$ disappears. Instead, $D_{4}$ waves are enhanced below $180 \mathrm{~m}$. The elevated $K E_{D 4}$ below $180 \mathrm{~m}$ occurs nearly synchronously with the enhanced subharmonics and near-surface elevation of $K E_{D 2}$ (Figure 3f). These significant variations of $D_{4}$ waves may be due to different nonlinear processes. On yeardays 246 to 248 , the 
(a)

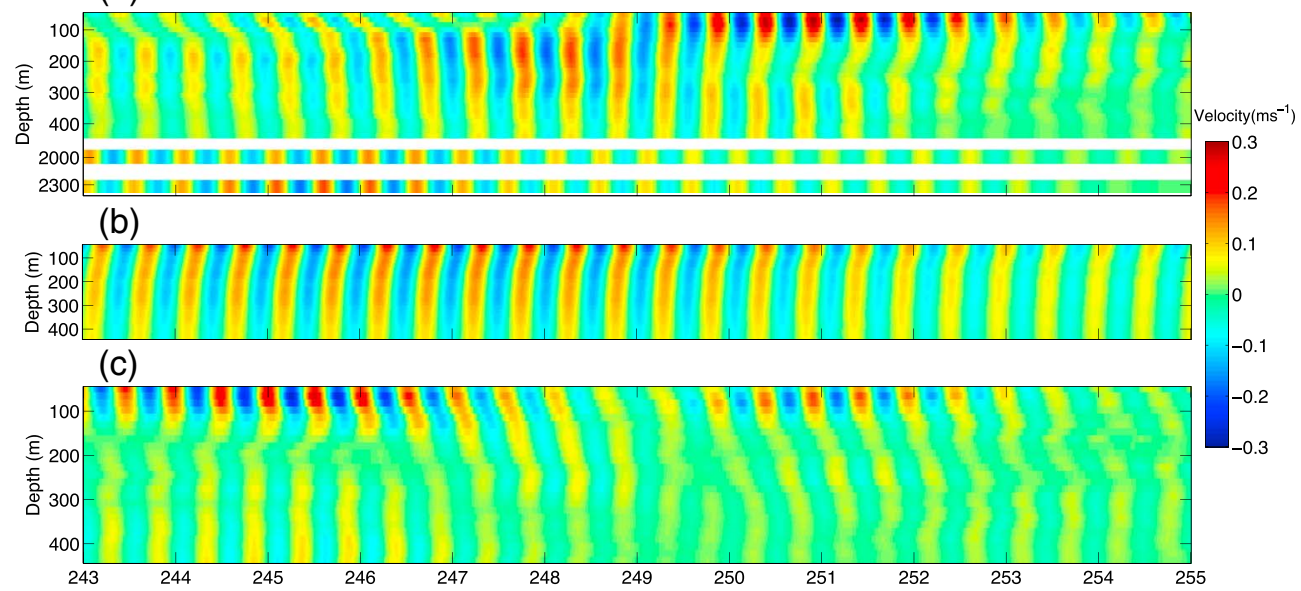

(d)
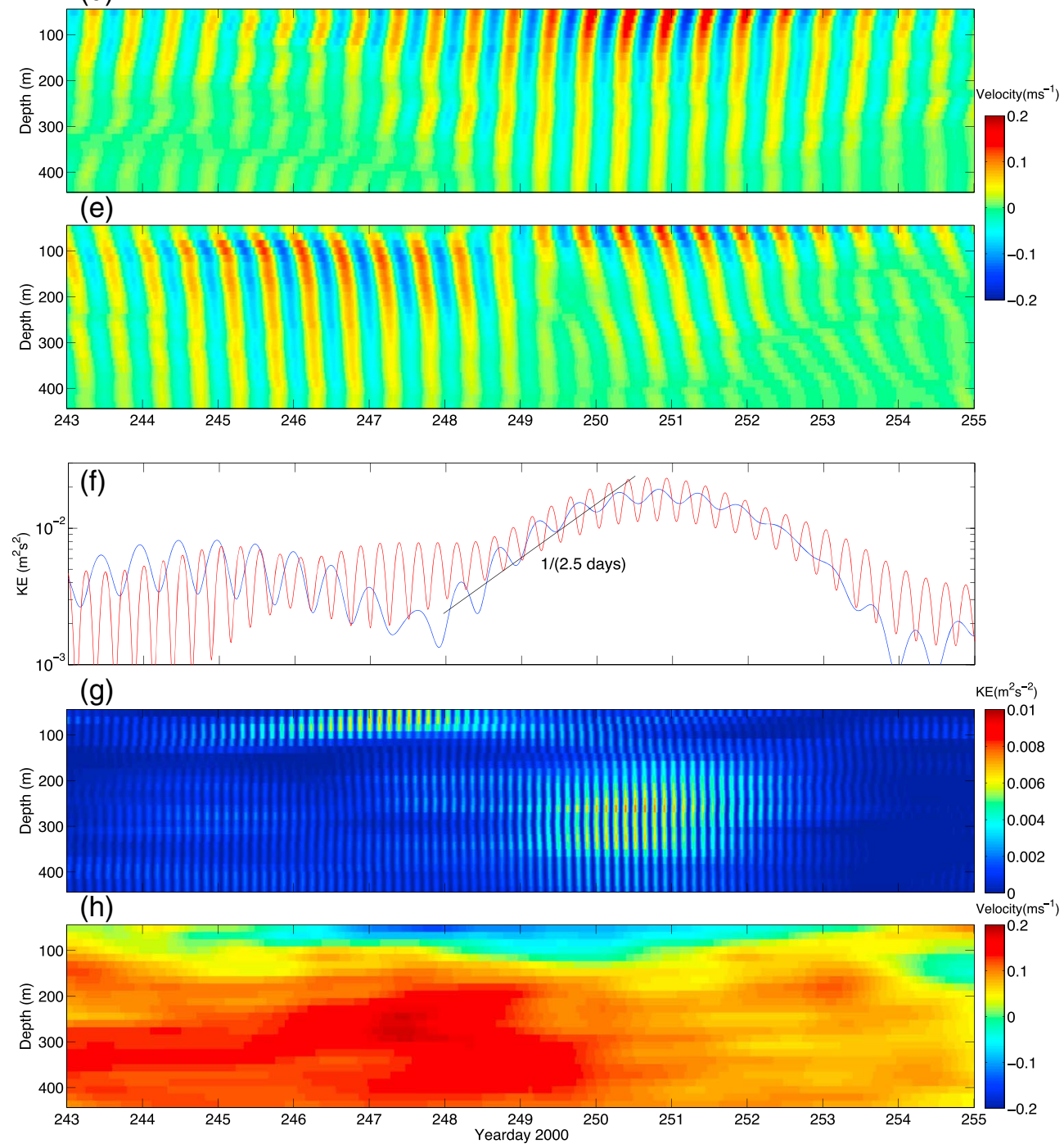

Figure 3. Time series of $K E$ and velocity on yeardays 241 to 260 . (a) Band-pass-filtered $D_{2}$ velocity. Values at 2000 and $2300 \mathrm{~m}$ are multiplied by a factor of 3. (b) Coherent $M_{2}+S_{2}$ and (c) $\mathrm{ICIT}_{D 2}+\mathrm{CIT}_{2 N 2}$ for the ADCP data only. (d) $\mathrm{CW}$ and (e) ACW motions of total $D_{2}$ signals from the ADCP data. (f) $K E_{D 2} / 3$ averaged over the depth bins above $120 \mathrm{~m}$ (red line) and $K E_{i c D 1}$ averaged over the depth range of $150-300 \mathrm{~m}$ (blue line). The oblique line indicates the subharmonic energy growth rate with $1 /\left(2.5\right.$ days). (g) $K E_{D 4}$. (h) The low-pass-filtered mean flow using the Butterworth filter with a cutoff frequency of 1/(3 days). Data in Figures 3a, 3b, 3c, and $3 \mathrm{~h}$ are computed for the zonal velocity. 

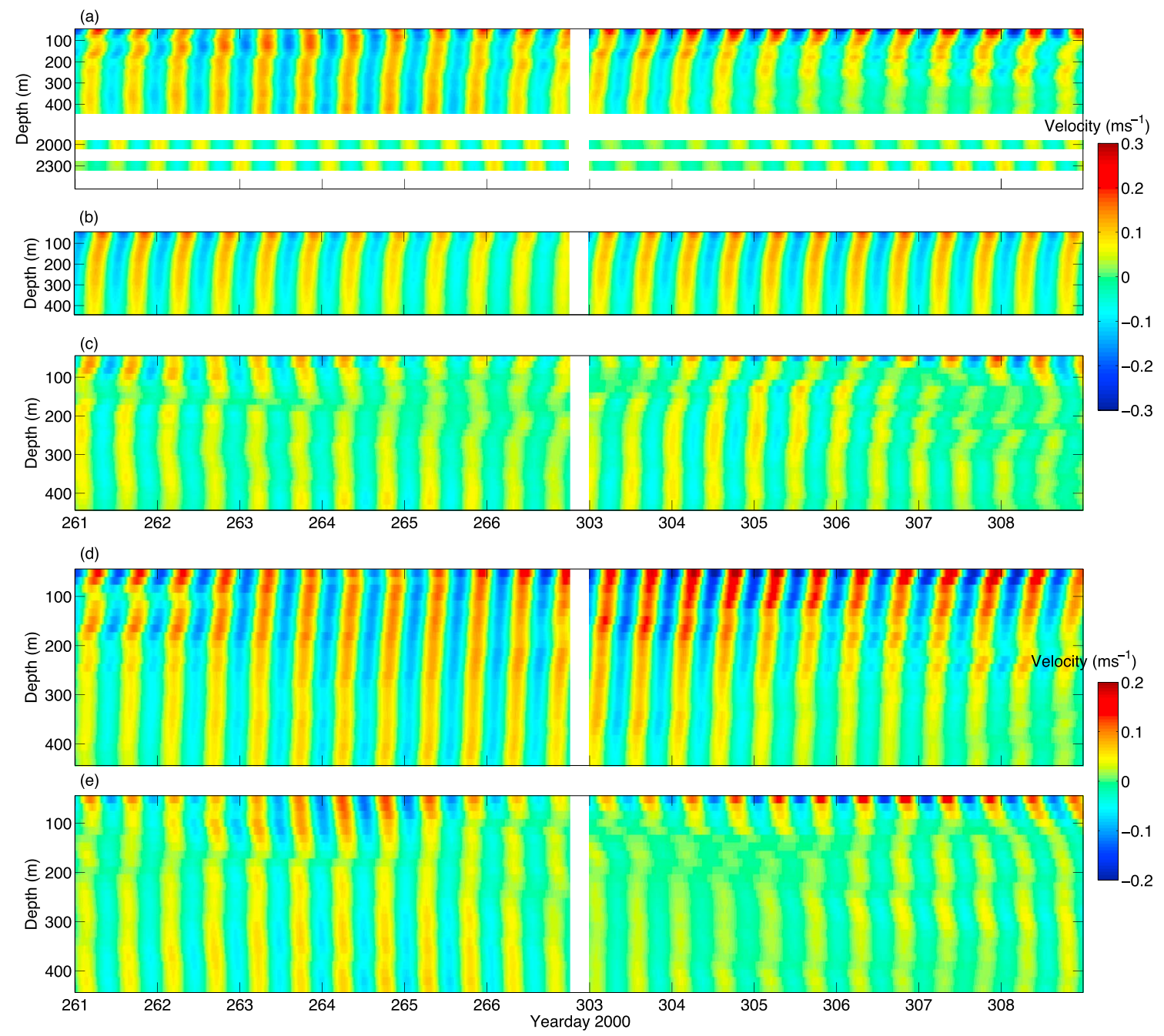

Figure 4. Similar to Figures 3a, 3b, 3c, 3d, and 3e but for the periods around SST-2 and SST-5.

generation of $D_{4}$ waves and the westward mean flow may be due to weakly nonlinear wave-wave interaction when the primary waves $\left(D_{2}\right)$ are relatively weak, as shown by Tabaei et al. [2005]. After yearday 248, strong subharmonics and $D_{4}$ waves are likely the result from strong nonlinear instability when the primary waves are enhanced near the surface. However, it is unclear why the depth range with the elevated $D_{4}$ waves is out of the range of near-surface intensification of $D_{2}$ waves on yeardays 249 to 252 .

[15] Two features of the mean flow, namely the confinement of the flow to the upper layer and the consistence of internal tidal horizontal propagation and the flow directions, agree with the laboratory results by Mercier et al. [2012]. So it seems that the surface reflection of $\mathrm{IT}_{D 2}$ may occur near our observation location, thereby generating strong subharmonics caused by PSI and a westward mean current. We note that Mercier et al. [2012] did not show the occurrence of PSI. This is probably because their experiments are without rotation, which plays an important role in improving subharmonic energy growth [MacKinnon and Winters, 2005].

\section{Summary}

[16] Current profiles have been presented suggesting the occurrence of pronounced nonlinear interactions in the region where two oppositely (upward and downward) traveling semidiurnal internal tidal waves encounter. Following numerical simulations by Korobov and Lamb [2008], our observations confirm that PSI of the IT can be effectively enhanced in the reflection of ITs from the boundary. There, PSI is likely to be a strong, rapid nonlinear instability, rather than the classic weakly nonlinear wave-wave interaction. In addition to subharmonics, a mean flow, along with higher harmonics, is also generated by the interaction between two vertically oppositely traveling waves. Although the presence of internal tidal beams cannot be confirmed from our current data, the theoretical framework by Tabaei et al. [2005] may be used to explain generation of higher harmonics and the mean flow. Their theory, however, does not explain the occurrence of strongly rapid PSI in the reflection area of internal waves. For the mean flow, some other interesting phenomena seen in the laboratory experiments by Mercier et al. [2012] cannot be identified due to the limitation of our data. For these, further study and more data are necessary.

[17] Acknowledgments. We thank Dongxiao Wang for the data used in this study. This work is supported by projects 2013CB430303, XDA10010104, U1033002 and 41276021.

[18] Meric A. Srokosz thanks two anonymous reviewers for their assistance in evaluating this paper. 


\section{XIE ET AL.: NONLINEAR INSTABILITY IN THE REFLECTION}

\section{References}

Alford, M. H., J. A. MacKinnon, Z. Zhao, R. Pinkel, J. Klymak, and T. Peacock (2007), Internal waves across the Pacific, Geophys. Res. Lett., 34, L24601, doi:10.1029/2007GL031566.

Carter, G. S., and M. C. Gregg (2006), Persistent near-diurnal internal waves observed above a site of M2 barotropic-to-baroclinic conversion, J. Phys. Oceanogr., 36, 1136-1147.

Emery, W. J., and R. E. Thomson (2001), Data analysis methods in physical oceanography, (second and revised edition), 638 pp., Elsevier Sci..

Gerkema, T., C. Staquet, and P. Bouruet-Aubertot (2006), Decay of semidiurnal internal-tide beams due to subharmonic resonance, Geophys. Res. Lett., 33, L08604, doi:10.1029/2005GL025105.

Hibiya, T., M. Nagasawa, and Y. Niwa (2002), Nonlinear energy transfer within the oceanic internal wave spectrum at mid and high latitudes, J. Geophys. Res., 107, 3207. doi:10.1029/2001JC001210.

Korobov, S. A., and K. Lamb (2008), Interharmonics in internal gravity waves generated by tide-topography interaction, J. Fluid Mech., 611, 61-95.

Javam, A., J. Imberger, and S. W. Armfield (2000), Numerical study of internal wave reflection from sloping boundaries, J. Fluid Mech., 396, 183-201.

Lamb, K. G. (2004), Nonlinear interaction among internal wave beams generated by tidal flow over supercritical topography, Geophys. Res. Lett., 31, L09313, doi:10.1029/2003GL019393.

Leaman, K., and T. Sanford (1975), Vertical energy propogation of internal waves: A vector spectral analysis of velocity profiles, J. Geophys. Res., 80, 1975-1978.

Leblond, P. H., and L. A. Mysak (1978), Waves in the Ocean, Elsevier, New York, pp. 602 .

Lien, R.-C., T. Y. Tang, M. H. Chang, and E. A. D’Asaro (2005), Energy of nonlinear internal waves in the South China Sea, Geophys. Res. Lett., 32 L05615, doi:10.1029/2004GL022012.

MacKinnon, J. A., and K. B. Winters (2005), Subtropical catastrophe: Significant loss of low-mode tidal energy at $28.9^{\circ} \mathrm{N}$, Geophys. Res. Lett., 32 , L15605, doi:10.1029/2005GL023376.
MacKinnon, J. A., M. Alford, O. M. Sun, R. Pinkel, Z. Zhao, and J. M Klymak (2013), Parametric subharmonic instability of the internal tide at $29^{\circ} \mathrm{N}$, J. Phys. Oceanogr., 43, 17-28.

McComas, C. H., and F. P. Bretherton (1977), Resonant interaction of oceanic internal waves, J. Geophys. Res., 82, 1397-1412.

Mercier, M. J., M. Mathur, L. Gostiaux, T. Gerkema, J. M. Magalhães, J. C. B. Da Silva, and T. Dauxois (2012), Soliton generation by internal tidal beams impinging on a pycnocline: Laboratory experiments, J. Fluid Mech., 704, 37-60.

Tabaei, A., T. R. Akylas, and K. G. Lamb (2005), Nonlinear effects in reflecting and colliding internal wave beams, J. Fluid Mech., 526, 217-243.

Teoh, S. G., G. N. Ivey, and J. Imberger (1997), Laboratory study of the interaction between two internal wave rays, J. Fluid Mech., 336, 91-122.

van Haren, H., L. Maas, and H. van Aken (2002), On the nature of internal wave spectra near a continental slope, Geophys. Res. Lett., 29, 1615. doi:10.1029/2001GL014341.

van Haren, H. (2004), Incoherent internal tidal currents in the deep ocean, Ocean Dyn., 54, 66-76. doi:10.1007/s10236-003-0083-2.

van Haren, H. (2005), Tidal and near-inertial peak variations around the diurnal critical latitude, Geophys. Res. Lett., 32, L23611, doi:10.1029/ 2005GL024160.

Xie, X.-H., G.-Y. Chen, X.-D. Shang, and W.-D. Fang (2008), Evolution of the semidiurnal $\left(M_{2}\right)$ internal tide on the continental slope of the northern South China Sea, Geophys. Res. Lett., 35, L13604, doi:10.1029/ 2008 GL034179.

Xie X.-H., X.-D. Shang, G.-Y. Chen, and L. Sun (2009), Variations of diurnal and inertial spectral peaks near the bi-diurnal critical latitude, Geophys. Res. Lett., 36, L02606, doi:10.1029/2008GL036383.

Xie, X.-H., X.-D. Shang, H. van Haren, G.-Y. Chen, and Y.-Z. Zhang (2011), Observations of parametric subharmonic instability-induced near-inertial waves equatorward of the critical diurnal latitude, Geophys. Res. Lett., 38, L05603, doi:10.1029/2010GL046521. 